\title{
Evacuación de feto muerto in útero, con sensibilización estrogénica previa. Informe preliminar.
}

\author{
Dr. Fernando Del Corral G. \\ Dr. Néstor H. Amorocho P.
}

De la Clínica de Maternidad de Cali - Valle

La muerte del feto in útero antes de la iniciación del trabajo de parto, se encuentra con alguna frecuencia en la práctica obstétrica, particularmente en nuestra clientela hospitalaria debido al escaso control prenatal.

La mayoría de las veces es imposible determinar el tiempo transcurrido desde la muerte fetal así como evaluar con exactitud el tiempo de su retención in útero.

Si bien es cierto que el $75 \%$ de los fetos muertos in útero son evacuados espontáneamente dentro de las dos primeras semanas (18), un $25 \%$ suelen retenerse hasta por 61 y más días después de su muerte. (18). Tanto en el primer caso como en el segundo, la muerte in útero puede acarrear serios problemas entre los cuales debemos mencionar: trastornos siquiátricos, alteraciones en los mecanismos de coagulación, infección, etc.

La muerte fetal es generalmente presentida angustiosamente por la paciente y aún a veces diagnosticada por ella misma. Los exámenes complementarios para su comprobación, la previenen aún más de la posibilidad, colocándola en un terreno muy poco benéfico para su estabilidad mental. Ciertos facultativos informan a su enferma de este hecho, mientras que otros solo hacen partícipe de dicho accidente al marido. En uno u otro caso, se hace para nosotros más ardua la tarea de estabilización emocional de los cónyuges, si es que se puede lograr una "estabilidad", siendo la madre la tumba viva de su hijo muerto. 
Nuevos sistemas de conducción del parto mediante el uso de la sico-profilaxis así como los acercamientos siquiátricos para combatir los trastornos de la hemorragia funcional y dismenorrea, son ejemplos de la importancia que tiene para el Ginecólogo de hoy en día, el manejo de su paciente a través de la esfera síquica, evitándole traumatismos causantes de un mayor "distress", ya de por sí tan prodigado en la vida moderna.

Por ello las escuelas obstétricas actuales tienden cada vez más a establecer normas y conductas encaminadas a suministrar un mejor "soporte" sicológico a las enfermas.

Dejando a un lado los problemas sicológicos que implica la muerte fetal, nos encontramos ante la presencia de un estado, que al prolongarse podría comprometer por otros aspectos el futuro inmediato de la paciente en mención.

Conocemos las observaciones hechas por Weiner, Reid, Roby y Diamond (1), desde 1950, sobre la relación existente entre la muerte fetal y su retención in útero con los cambios en los mecanismos de coagulación que predisponen a serias hemorragias. Estos trastornos son tanto más severos si la causa de la muerte fetal es la incompatibilidad por $\mathrm{Rh}$ con sensibilización materna (1).

La corrección causal de dichas hemorragias, las cuales, como es sabido son la mayor parte de las veces debidas a bajas en los niveles circulantes de fibrinógeno, traen consigo frecuentemente serios problemas consecuentes a incompatibilidad de grupos 0 sub-grupos o a la indeseada aparición de hepatitis por suero homólogo.

Desde hace poco tiempo hemos iniciado una conducta más radical en el Departamento de Obstetricia de la Universidad del Valle, dirigida a evacuar el útero mediante inducción médica $\mathrm{y}$ quirúrgica, previa sensibilización con estrógenos conjugados.* Sobre este punto existen grandes controversias. Así por ejemplo: Smith (23), en 1908 basándose primordialmente en los problemas de "ansiedad", aconsejaba la evacuación inmediata del útero, tan pronto como se hiciere con certidumbre, el diagnóstico de

* Ayerogen intravenoso de Ayerst Laboratories. New York. 
muierte fetal. Contempló además la posibilidad de muerte materna causada por "la absorción de toxinas fetales". Dippel (18), más tarde solo aconseja la evacuación uterina asociada a otros problemas secundarios, particularmente cuando la muerte fetal per se, ocasiona una perturbación mental para la madre.

Tricomi y Kohl (2), en una de sus recientes publicaciones enfáticamente desaconsejan cualquier interferencia que tenga por objeto evacuar los productos de la concepción de un útero, auncuando se haya demostrado defectos en los mecanismos de coagulación. Por el contrario, Pritchard (3), aconseja evacuar el útero después de la tercera semana de retención, tiempo desde el cual comienzan a presentarse los trastornos en los mecanismos de coagulación o los estados severos de ansiedad.

Una de las objeciones principales que en general existen sobre inducción es la falta de respuesta, la que en algunos casos implica intervenciones de mayor magnitud (3). Creemos que con el uso de los estrógenos pueden obviarse algunos de estos problemas y por ello hemos querido presentar los casos siguientes:

\section{INDICACIONES :}

Para estos estudios hemos considerado como indicación para la inducción, la muerte fetal uterina por encima de las 16 semanas, causada por Toxemia, incompatibilidad por Rh, Abruptio placentae, Diabetes, Sífilis, circulares del cordón, así como las muertes intra-uterinas de etiología desconocida, una vez que por medios clínicos y radiológicos se haya establecido el diagnóstico durante la primera semana de observación.

\section{CONDICIONES PARA LA INDUCCION:}

Nos hemos ceñido a las normas establecidas para toda inducción en general, sin tener en cuenta el estado de madurez y localización del cuello. (Clasificados de acuerdo a lo propuesto por Cocks 6 y Nixon 7).

\section{TECNICA DE INDUCCION:}

Sensibitización: - Eștrógenos conjugados (Ayerogen) 40 miligramos administrados en la siguiente forma: 20 miligramos 
vía endovenosa 12 horas antes de comenzar la infusión ocitócica y una segunda dosis de 20 miligramos vía endovenosa 6 horas después de la primera.

\section{OCITOCICO :}

Ocitocina sintética (Syntominon?) 5 U. I. en 500 c.c. de vehículo (Dextrosa al $5 \%$ en agua o salina, de acuerdo con el caso). Cuando la solución salina no estaba contraindicada, se prefirió ésta como vehículo.

El método usado para inducción fué el descrito por Caldeyro y Alvarez (4), en el cual dos gotas de la infusión corresponden a una miliunidad de agente ocitócico.

Cuando más de una inducción fue necesaria, ésta se hizo en un período no inferior a 6 horas de la inducción precedente. En este caso la segunda inducción se inició con una dosis igual a la última usada en la inducción anterior, y así sucesivamente.

\section{RUPTURA DE MEMBRANAS}

Si la respuesta a la inducción era favorable (apreciación clínica y tocográfica $\stackrel{0}{)}$, tan pronto como las condiciones del cuello lo permitieron, se practicó amniotomía artificial por los procedimientos corrientes.

\section{CONTROLES DE LA INDUCCION:}

Los controles de la inducción se hicieron clínicamente por su respuesta sobre TA, pulso y respiración. En algunos casos se practicaron registros tocográficos y electro-cardiográficos, antes y durante la administración de Syntocinon. La velocidad de la inducción fue regulada mediante la valoración clínica y tocográfica o aumentándose o disminuyéndose de acuerdo con la frecuencia e intensidad de las contracciones. Debemos anotar que sobre este

Syntocinon. Suministrado por Pharmaceutical Division of the Sandoz Chemical Company. Hanover N J. Sintetizado por el método de Boissonnas. (5).

- El Tocógrafo usado fue el de Lorand, modelo 1958. 
particular no existen normas establecidas ya que el comportamiento uterino a los ocitócicos es variable, según la enferma, dependiendo además del estado postural de la misma.

\section{CONDUCCION DEL PARTO:}

En los casos que a continuación presentamos, dos tuvieron parto espontáneo y en tres casos $(1-4$ y 5$)$ se hicieron maniobras de Rojas y Mauriceau; fórceps de Piper; y gran extracción podálica, respectivamente.

\section{CASO No 1}

H. Cl. No 73243 .

G. M. de F. 38 años.

Fecha de ingreso: Julio 1/60, 17:30 horas.

Causa de Ingreso: Grávida 7 para 5. Embarazo de más o menos 29 semanas, feto muerto retenido.

Antecedentes: Primer parto feto vivo, normal a término; segundo parto a término, feto vivo, normal tercero; parto a término, feto vivo ictérico que murió en pocos minutos. Tres embarazos subsiguientes partos inducidos por feto muerto entre las 26 y 30 semanas; sexto embarazo, parto a las 36 semanas, feto hidrópico muerto desde las 32 . En este embarazo no percibe movimientos fetales desde hace 15 días.

Examen físico: T. A. 120/80, Pulso 92 por minuto, Temperatura $37.3^{\circ}$ C.

Obstétrico-Ginecológico: Utero 25 ctms. de altura. Ultima regla Nov. 25/59. Cuello uterino tipo 2.

Evolución: Julio 2/60, 7:00 horas. Previa aplicación de Ayerogen se inicia la inducción (13:30 horas de su hospitalización). Respuesta a los 40 minutos.

Julio 2/60, 17:00 horas amniotomía, se inicia segunda inducción.

Duración del primer período: 6 horas.

Duración del segundo período: 0:30 horas.

Duración del tercer período: 0:05 horas.

Duración total: $6: 35$ horas.

Parto espontáneo. Causa de muerte fetal: Eritroblastosis (madre Rh-) Peso del feto: 850 grms., macerado e hidrópico. Pérdida de sangre, 250 c.c.

\section{CASO No 2}

H. Cl. 107-10-59.

A. E. de G. 38 años.

Fecha de ingreso: Octubre $3 / 59$. 
Causa de ingreso: Grávida 10 para 4. Embarazo de 20 semanas. Feto muerto retenido.

Antecedentes: Un parto prematuro con feto muerto; un mortinato; tres abortos.

Examen Físico: T. A. 120/80. Pulso 80. Febril.

Obstétrico-Ginecológico: Utero para 20 semanas de embarazo. Ultima regla Mayo 25/59. Cuello uterino tipo 3.

Evolución: Tuvo tres inducciones así:

1a) Octubre $3 / 59$ - 24:00 horas 10 unidades Pitocín, en 500 c.c. de Dextrosa al $5 \%$ en A. D. Dosis hasta $32 \mathrm{~m}$. un./minuto.

2a) Octubre 4/59 - 13:00 horas. Ocitócico, vehículo y dosis igual a la anterior.

$3^{\text {a) }}$ Octubre 5/59 - 5:15 horas. 20 unidades Pitocín, en 500 c.c. de Dextrosa al $5 \%$ en A. D. Dosis hasta $120 \mathrm{~m}$. un./minuto.

Previa aplicación de Ayerogen, se hace nueva inducción con Syntocinon.

Octubre 10/59 - 16:00 horas. Se inicia inducción con $120 \mathrm{~m}$. un./minuto. Hace aborto incompleto a las 17:40 horas, lográndose efecto deseado en 1:40 horas.

\section{CASO No 3}

M. V. 30 años.

H. Cl. No $911-10-59$.

Fecha de Ingreso: Octubre $30 / 59$.

Causa de ingreso: Grávida 7 para 5. Embarazo de 34 semanas. Feto único muerto.

Presentación Pelvis.

Antecedentes Obstétricos: $5^{\circ}$ embarazo, aborto de 14 semanas; $6^{\circ}$ embarazo, parto a las 32 semanas, feto muerto macerado.

Examen Físico: T. A. 90/65. Pulso 80. Serología positiva.

Obstétrico-Ginecológico: Altura uterina $27 \mathrm{cms}$. Ultima regla Febrero 28/59. Cuello uterino tipo 2. Membranas íntegras. Presentación Pelvis. En control prenatal se empezó a tratar la Lues en Octubre $2 / 59$; ausencia de movimientos fetales, en Octubre 20/59. 
Evolución: Noviembre 6/59 - 23:00 horas. Previa aplicación Ayerogen. se inicia inducción.

Duración del Primer Período: 12:40 horas.

Duración del Segundo Período: 0:30 horas.

Duración del Tercer Período: 0:10 horas.

Duración total: 13:20 horas.

Parto intervenido por Fórceps de Piper. Causa de muerte fetal: Lues. Peso del niño 2.490 grs. Pérdida de sangre 200 c.c.

\section{CASO No 4}

H. Cl. No 167-11-59.

E. J. R. 22 años.

Fecha de Ingreso: Noviembre 6/59 - 11:45 horas. muerto.

Causa de Ingreso: Grávida 2 para 1. Embarazo de 31 semanas. Feto único

Examen Físico: T. A. 110/60. Pulso 80.

Obstétrico-Ginecológico: Altura uterina $27 \mathrm{cms}$. Ultima regla primeros días Abril/59. Cuello uterino tipo 2. No contracciones uterinas. Presentación pelvis incompleta.

Evolución: Noviembre 7/59 - 10:00 horas. (Previa aplicación Ayerogen). Se inicia inducción.

Duración del Primer Período: 9:30 horas.

Duración del Segundo Período: 0:15 horas.

Duración del Tercer Período: 0:00 horas.

Duración total: 9:45 horas.

Parto intervenido por maniobras de Rojas y Mauriceau. Causa de muerte ietal: Desprendimiento placentario sin signos clínicos; $3 / 4$ partes de la placenta estaban cubiertas por coágulos oscuros y signos placentarios evidentes de desprendimiento.

Peso del Niño 1.360 grs. Pérdida de sangre 300 c.c

\section{CASO No 5}

H. Cl. No $549-11-59$.

R. E. C. 30 años.

Fecha de Ingreso: Noviembre 18/59. - 14:45 horas.

Causa de Ingreso: Grávida 7 para 6. Embarazo de 39 semanas. Feto único muerto. 
Antecedentes de Diabetes. Diabetes comprobada por curvas de tolerancia a la glucosa.

Antecedentes de fetos gigantes. Primer embarazo, mortinato; Quinto embarazo, parto prematuro.

Examen Físico: T. A. 110/70. Pulso 80.

Obstétrico-Ginecológico: Altura uterina $32 \mathrm{cms}$. Ultima regla Febrero 18/59. Cuello uterino tipo 2. No hay contracciones uterinas. Presentación cefálica.

Evolución: Noviembre 20/59 - 9:35 horas. (Previa aplicación Ayerogen se inicia inducción; respuesta a los 15 minutos, contracciones inefectivas. (Figura $1-\mathrm{A})$.
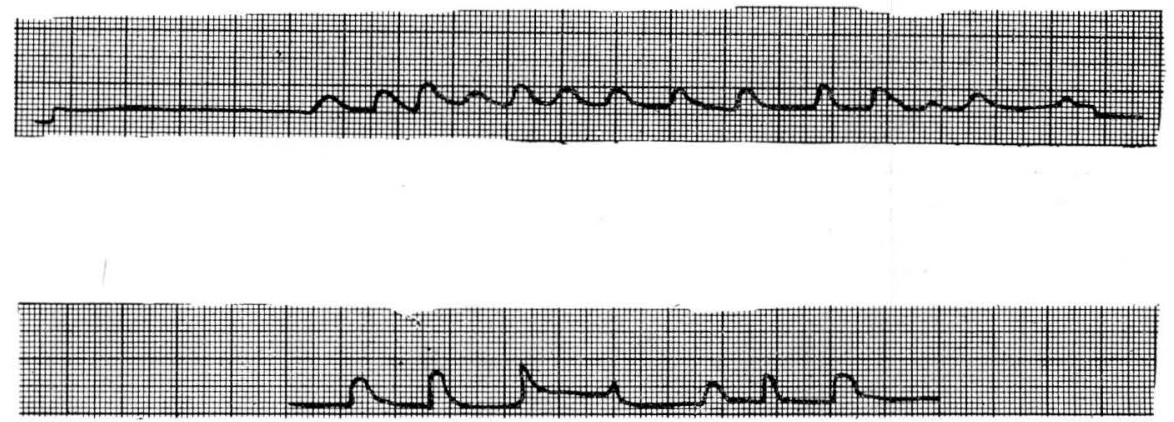

FIGURA No 1 - A - Gráfica tomada al comienzo de la primera inducción, previa sensibilización con Ayerogen. Respuesta a los 15 minutos con contracciones de Braxton Hicks, frecuentes y regulares. No se observó progreso en la dilatación cervical durante esta inducción.

B - Segunda inducción 24 horas más tarde. Se observan contracciones uterinas intensas y frecuentes, efectivas para producir el parto en 4:30 horas. (16 mili-unidades/minuto).

Noviembre 21/59 - 9:15 horas. Iniciación segunda inducción (Fig. 1-B).

Duración del Primer Período: 4:15 horas.

Duración del Segundo Período: 0:15 horas. (Ruptura espontánea de memb. con 3 cms.).

Duración del Tercer Período: 0:15 horas.

Duración total: 4:45 horas.

Parto espontáneo. Causa de muerte fetal: Insuficiencia placentaria por diabetes. Peso del Niño 3.570 grs. Pérdida de sangre 300 c.c.

\section{COMENTARIO}

Por muchos años se ha puesto en duda el efecto que los estrógenos puedan tener como sensibilizantes de la fibra uterina. 
Las experiencias iniciales basadas en trabajos en animales in vitro, especialmente, (8) demostraron la actividad de los estrógenos sobre la fibra uterina, los cuales producen contracciones amplias e infrecuentes. Reynolds y Friedman, corroboraron los hallazgos de Keye demostrando que el útero se presentaba muy activo, cuando estaba influenciado por los estrógenos. (9)

Fueron los trabajos de Csapo, los que vinieron a demostrar cómo los estrógenos actuaban sobre la contracción uterina, aumentando las proteínas contráctiles y acelerando las combustiones necesarias para dicha contracción. Estos mecanismos mencionados ya fueron anotados en comunicación precedente por uno de nosotros (F. del C.) (22).

Los estrógenos por lo tanto, son la hormona principal necesaria para la síntesis de todo el sistema contráctil del miometrio, así como para el mantenimiento de contracciones rítímicas y espontáneas. (10) Los estudios clínicos realizados con ellos han sido en muchas ocasiones, base de conjetura y han hecho que muchos especialistas no los usen o los desaconsejen como sensibilizantes de la fibra.

Por otra parte, la inducción con mejores procedimientos de control y el uso de drogas más seguras (Ocitócicos sin acción vasopresora $\left.{ }^{\circ}\right)$, han ido tomando en la práctica actual cada vez más incremento. La mayor parte de los fracasos en la inducción electiva o terapéutica son atribuíbles generalmente, a un manejo inadecuado de los ocitócicos, al no ser usados en dosis progresivas controladas, (4) de acuerdo a la respuesta clínica y tocográfica. Hoy día sabemos mediante estudios electro-histerográficos recientes, que las contracciones inducidas por la ocitocina, son fisiológicamente idénticas a las logradas en un trabajo de parto normal por la ocitocina endógena. (11) Si bien es cierto que no solamente la ocitocina es la única responsable de la iniciación de un trabajo de parto, sí sabemos que juega un papel preponderante.

Creemos que con el uso de estrógenos (Ayerogen) para sensibilizar la fibra uterina previos a la aplicación de Ocitocina ${ }^{0}$ se puede manejar el problema de feto muerto in útero y posiblemente los fracasos sean menores que cuando se usa el Ocitócico solo.

\footnotetext{
o Syntocinon.

o Syntocinon.
} 
En los cinco casos inducidos, con sensibilización previa, no tuvimos ningún fracaso y con las dosis usadas del ocitócico, no se presentó ninguna complicación, ni efectos secundarios.

Creemos que el Syntocinon es un buen ocitócico y en nuestros casos no observamos modificaciones apreciables sobre tensión arterial, pulso y respiración, factores éstos ya anotados anteriormente $(13,14,15,16,17)$. No obstante, en otros estudios, estas modificaciones han sido comunicadas, pero nunca tan marcadas como las obtenidas con el Pitocín (19, y 20). En algunas ocasiones se han mencionado a su vez efectos depresores sobre el miocardio, con el uso de los ocitócicos y así por ejemplo Mayes y Shearman (21), encontraron aplanamiento o inversión de la onda $\mathrm{T}$ al E. C. G. mediante el uso del Syntocinon. Mayes encontró que en tres de cuatro sujetos se producían alteraciones similares y cita a su vez a Sjostrand, quien observó el mismo fenómeno.

Nosotros en los tres casos con E. C. G., tomados antes y durante la administración de Syntocinon, encontramos similares hallazgos durante la infusión en el caso $N^{0}$. 5 . Parece que dichas alteraciones sean debidas más que a un espasmo coronario, a una interferencia en el metabolismo del miocardio (2). Fig. 2.

Algunos han considerado como contraindicaciones para la inducción, la presencia de un cuello inmaduro, pero nosotros creemos junto con Tamis y Shey (12), que aún en estos casos es posible repetir la inducción dos y tres veces, sin considerarla fallida; las inducciones precedentes servirán en tal caso para preparar favorablemente el cérvix.

Coincidencialmente en los cinco casos inducidos, tanto los moldeamientos como los cabalgamentos fetales no fueron severos para practicar embriotomías cefálicas, no obstante nos parece que en tales casos, sin tener en cuenta cuales sean las condiciones de la cabeza deben hacerse embriotomías, llenando los requisitos reglamentarios.

Tampoco tuvimos problemas de cuello, en los cuales está indicada la tracción del cuero cabelludo con el Clamp de Willet.

A pesar de que se ha encontrado una mayor pérdida sanguínea en el alumbramiento en los partos inducidos con ocitocina, nosotros no observamos hemorragia en los casos presentados. 

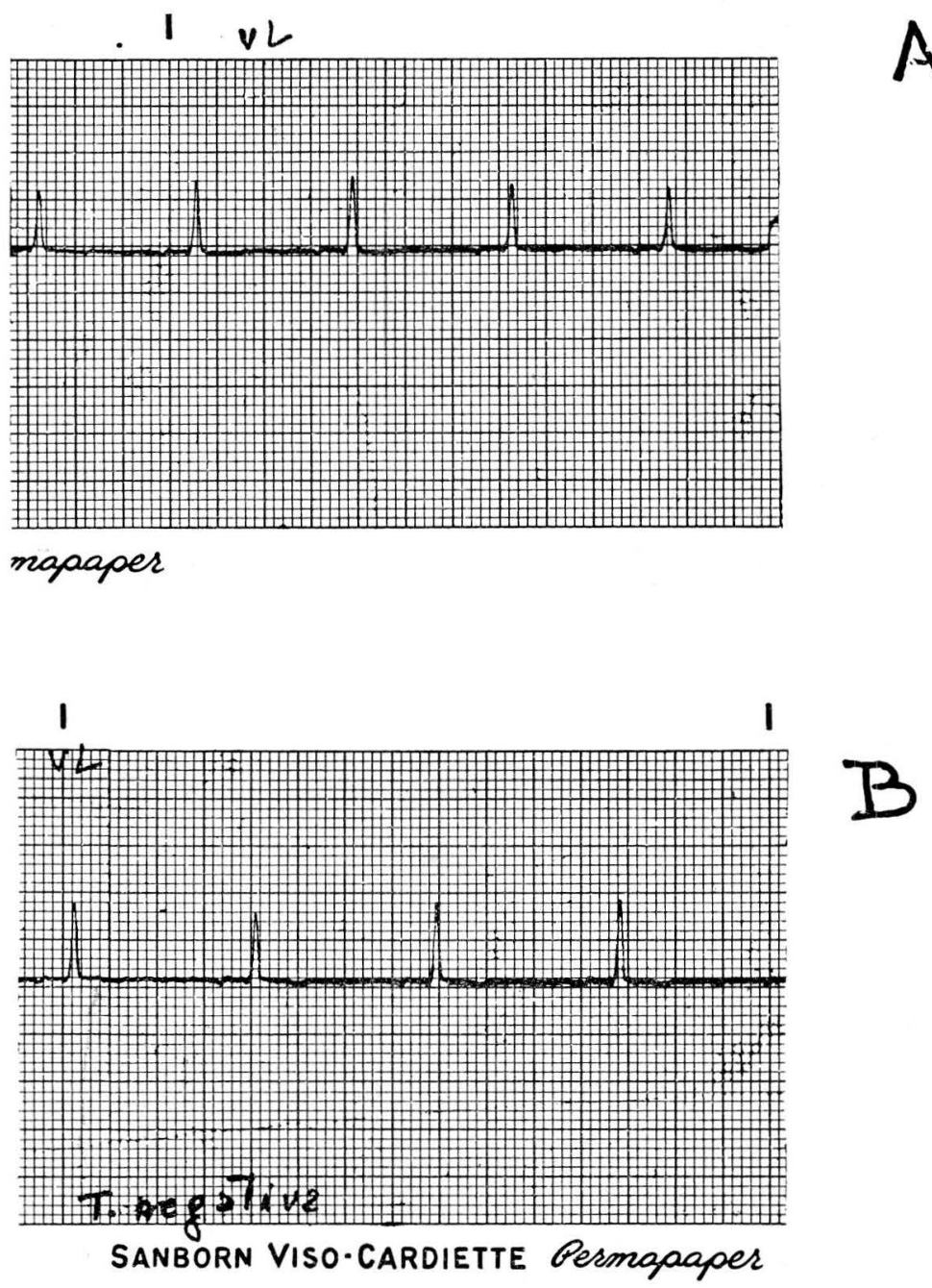

FIGURA No 2 - A - E. C. G.; derivación VL tomada antes de la inducción. B - E. C. G.; derivación VL. Durante la inducción (32 miliunidades/minuto) Nótese la presencia de la onda $T$ negativa, que se interpretó como isquemia subepicárdica lateral alta.

Dejamos a consideración de ustedes el manejo de los casos de feto muerto "in útero", con sensibilización previa estrogénica seguida de inducción, para una posterior valoración con un mayor número de casos. 


\section{RESUMEN}

Se presentan cinco casos de feto muerto "in útero", de la Clínica de Maternidad de Cali, tratados mediante sensibilización estrogénica previa seguida de inducción por infusión de ocitocina (Syntocinon) vía endovenosa.

Las cinco pacientes fueron multíparas y sus edades fluctuaron entre los 22 y los 38 años.

Todas presentaban un feto muerto in útero, entre las 20 a 40 semanas de embarazo: Las causas de muerte intrauterina fueron: eritroblastosis (Rh-), desprendimiento placentario, diabetes, sífilis y un aborto frustro del cual desconocemos la causa. Los cinco casos fueron inducidos con Syntocinon, en el curso de la primera semana de hospitalización.

De ellos dos respondieron en la primera inducción, dos requirieron dos inducciones (Caso $\mathrm{N}^{0}$ 1) y el último sólo respondió a la cuarta inducción con Syntocinon y estrógenos, después de haber fracasado en tres inducciones previas hechas con Pitocín y sin estrógenos. La duración total del trabajo fluctuó entre 1:40 y 13:20 horas, con un promedio aproximado de siete horas.

Las condiciones del cuello antes de inducción, fueron: tres pacientes con tipo 2, una con tipo 4 y la restante con tipo 3 de la clasificación de Cocks.

El alumbramiento fue espontáneo en los cinco partos.

La sangre perdida no sobrepasó los 300 c.c.

No hubo ninguna complicación en los casos presentados.

RECONOCIMIENTO: Agradecemos al Dr. Rafael Rodríguez F. Ex-presidente de la Clínica de Maternidad de Cali 1957-59, por la ayuda prestada en la realización de este trabajo al Dr. Alberto Naranjo por la lectura de los electrocardiogramas así como por su colaboración en la valoración cardio-pulmonar de estas pacientes.

\section{REFERENCIAS}

1 WEINER A. E., REID D. E., ROBY Ch. D., DIAMOND L. K. Coagulation defects with intrauterine death from $\mathrm{Rh}$ isosensitization. Am. J. Obst. \& Gynec. 60: 1015-1021 (Noviembre) 1950. 
2 TRICOMI, V., KOHL S. G. Fetal death in utero. Am. J. Obst. \& Gynec. 74: 1092 - 1957.

3 PRITCHARD J. A. Fetal death in utero. Obst. and Gynec. 14: 5, 573. 1959.

4 CALDEYRO BARCIA, ALVAREZ H., POSEIRO J., SICA BLANCO Y. CARBALLO M., MENDES-BAUER C., GONZALEZ PANIZZA, FIELITZ C. "Juicio crítico y resultados de la inducción y conducción del parto". Relato oficial presentado al III Congreso LatinoAmericano de Obstetricia y Ginecología y III Congreso Mexicano de Obstetricia y Ginecología.

5 BOISSONNAS R. A., et al: Helvet. chim. acta 38:1491, 1955.

6 COCKS, D. P.: Brit. M. J. 1: 327, 1955.

7 NIXON W. C. W. y SMYTH C. N. "Old and new methods for induction of labor and premature labor". Am. J. Obst. \& Gynec. 77: 343

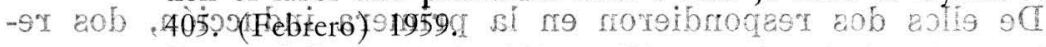

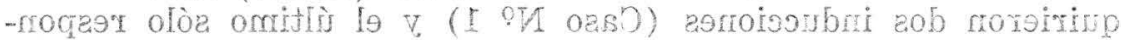

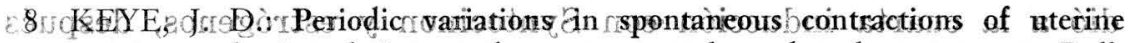
- ofic riog muscle, in retation to the oestreus cycle and early pregnancy Bull. oxjng onjohns Hopkins, Hospital. 34:60-63 (Febrero) 1923 . -95 REYNOLDS, S. R. M. and FRIEDMAN, M. H.: Studies on the uterus. III Activity of uterine fistula in unanesthetized rabbits following

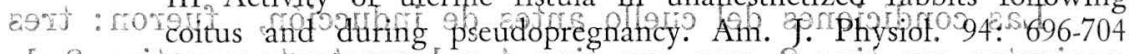

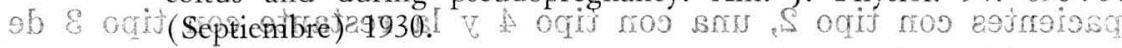
axioo ob nòiosolitiesio sil

10 "Clinical measurements of uterine forceps in pregnancy and labor". Rey. conolds S. R.oM.oy asociados̀. Charkes se. Thomas. Springfield Illinois. 1954.

.9 .9008 aol òsuordoa or sbibrod grense sut

11 "Electrohisterography". Larks S. D. Charles C. Thomas. Editado por Hamblen E. C. 1950.

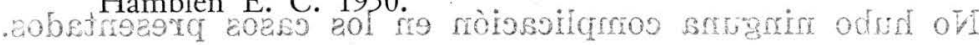

12 TAMIS B. A., SHEY A. I. Intravenogs Pitocin and the unripe cervix

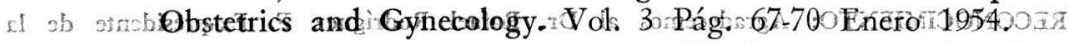

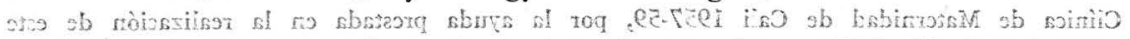

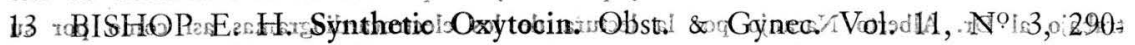

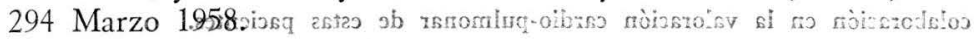

14 BOSCH K., KASEN O. La oxitocina enstetricia. Journal Suisse de Médicine. 86, 229-233 (1956, N⿳9).

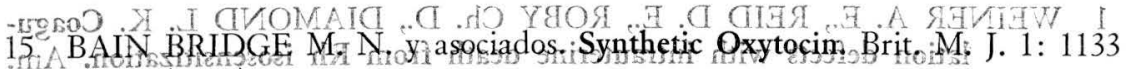

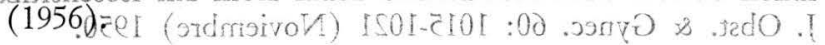


16 MORARI, M. Ertahrongen mit dem wehrmittel ots 68. Wien. med Wchnschr. 106: 458 (1956).

17 VELIKAY, L. Zur Verwendung eines Synthetischen Oxitocin in der medikamentosen Geburtsleintung. Zentralbl. Gynak 79: 846 (1957).

18 DIPPEL, A. L. Death of foetus in utero. Johns-hopkins. Bull. 54-24-47. 1934.

19 KONZETT H. BERDE B. CERLETTI A. El Syntocinon, hormona uterotónica del lóbulo posterior de la hipófisis. Journal Suisse de Médicine. 86, 226-229 (1956 No 9).

20 LUBY R. J. GRIER M. E., KEITGES P. W. TAYLOR R. J. Clinical Experience with A. Sinthetic Oxytocin (Syntocinon). Am. J. of Obst. \& Gynec. Vol. 77 No 1, 50-54 (Enero) 1959.

21 MAYES B. T. SHEARMAN R. B Experiense with Synthetic Oxytocin: The effects on the Cardiovascular system and its use for the insh srul daction of rabor and controf of the third Stage. ournal of Obst. \&

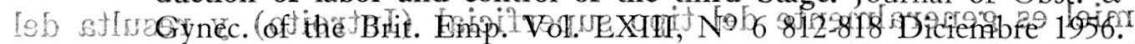

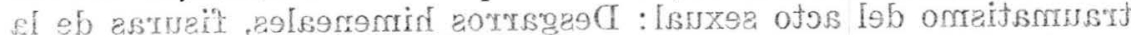

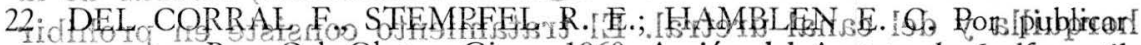
en Rev. Col. Obst . Gine 1960 . Acción del Acetato de 6 alfa-metil 17-alfa-Hidroxiprogesterona (provera) sobre las contracciones de Braxton-Hicks en el embarazo avanzado y algunos estudios sobre

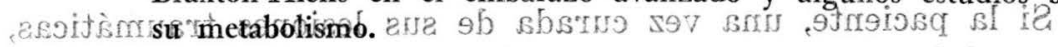

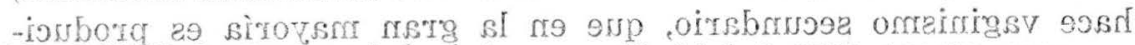

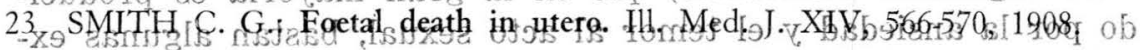

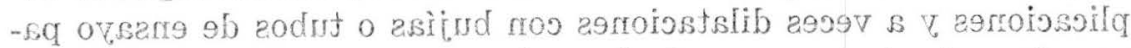

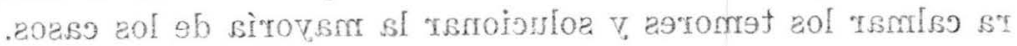

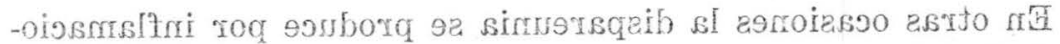

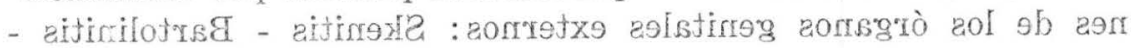

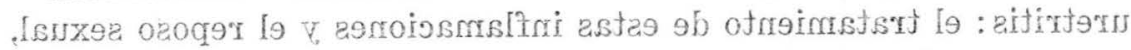

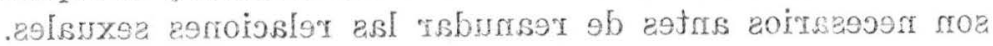

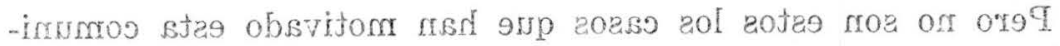

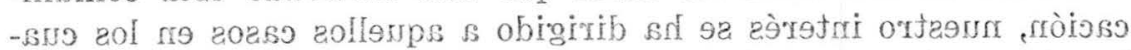

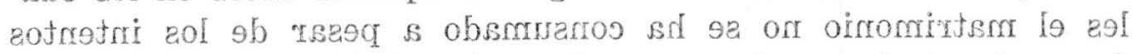

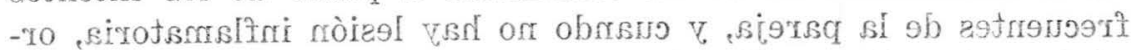
ronugls robsesq $\mathrm{V}$ sbiqmi of sup suugls ròiosmrotsm in soirs̀g .oinominsers l95 29e9m

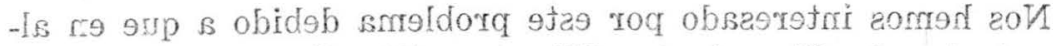
razeord V rozzorb (S) notranW : sigologerio 